\title{
Estado de la investigación en energía en Colombia: una mirada desde los grupos de investigación
}

\author{
Francy Nelly Jiménez-García ${ }^{1}$ \\ Alejandra María Restrepo-Franco ${ }^{2}$ \\ Luis Fernando Mulcue-Nieto ${ }^{3}$
}

Fecha de recepción: 10 de abril de 2019

Fecha de aceptación: 7 de junio de 2019

\section{Resumen}

En este artículo se presenta el estado de la investigación en Colombia en temas de energía, a partir de la revisión, la sistematización y el análisis de los resultados de los grupos de investigación. La información se obtuvo desde la plataforma Scienti de Colciencias, de una muestra de grupos de investigación que declaran tener líneas de interés en energía. Se tuvieron en cuenta tres categorías para el análisis de la información: ubicación geográfica de los grupos y su clasificación, intereses investigativos determinados desde las líneas de investigación propuestas, y producción científica de los grupos. Se encontró que el $75 \%$ de los grupos se ubica en cuatro de los 32 departamentos del país y que el $90 \%$ pertenece a instituciones de educación superior. Hay diversidad en cuanto a los intereses investigativos y se destacan cuatro líneas principales: energía y potencia, gestión energética, hidrocarburos y energías renovables. La producción científica está centrada básicamente en artículos científicos, libros y desarrollo de proyectos; en cuanto a patentes, los resultados son escasos. En relación con energías renovables, la producción científica empezó a surgir a partir de 1999 y ha experimentado un crecimiento con tendencia lineal. Se logró identificar que, si bien hay una investigación establecida en temas de energía desde las instituciones de educación superior y algunas empresas del sector energético, los avances relacionados con

\footnotetext{
${ }^{1}$ Ph. D. Universidad Autónoma de Manizales (Manizales-Caldas, Colombia). francy@autonoma.edu.co. ORCID: 0000-0003-1546-8426.

2 Universidad Autónoma de Manizales (Manizales-Caldas, Colombia). ORCID: 0000-0002-8173-7906.

${ }^{3}$ Ph. D. Universidad Autónoma de Manizales (Manizales-Caldas, Colombia). luisf.mulcuen@autonoma.edu.co. ORCID: $\underline{0000-0002-1544-3037 .}$.
} 
energías renovables son aún incipientes; hay un camino por recorrer, el cual se ha ido abonando con las nuevas reglamentaciones que se han dado en el país.

Palabras clave: energía; energías renovables; grupos de investigación; producción científica.

\section{The State of Energy Research in Colombia: A View from the Research Groups}

\section{Abstract}

This article presents the state of research in Colombia on energy issues from the review, systematization and analysis of the results of the research groups. The information was obtained from the Scienti platform of Colciencias of a sample of research groups that claim to have lines of interest in energy. Three categories were considered for the analysis of the information: geographical location of the groups and their classification; research interests determined from the proposed lines of research; and scientific production of the groups. It was found that $75 \%$ of the groups are in 4 of the 32 departments of the country and $90 \%$ belong to higher education institutions. There is diversity in terms of research interests, with four main lines of action: energy and power, energy management, hydrocarbons and renewable energies. The scientific production is centered basically in scientific articles, books and development of projects, as for patents the results are scarce. In relation to ER, the scientific production began to emerge from 1999 and has experienced a linear growth. It is possible to identify that although there is established research on energy issues from higher education institutions and some companies in the energy sector, advances related to renewable energies are still incipient; there is a road ahead which is fertilized with the new regulations that have been given in the country.

Keywords: energy; renewable energies; research groups; scientific production. 


\section{Estado da pesquisa em energia na Colômbia: uma visão desde os grupos de pesquisa}

\section{Resumo}

Neste artigo apresenta-se o estado da pesquisa na Colômbia em temas de energia, a partir da revisão, da sistematização e da análise dos resultados dos grupos de pesquisa. A informação obteve-se desde a plataforma Scienti de Colciencias, de uma amostra de grupos de pesquisa que declaram ter linhas de interesse em energia. Foram consideradas três categorias para a análise da informação: localização geográfica dos grupos e sua classificação, interesses de pesquisa determinados desde as linhas de pesquisa propostas, e produção científica dos grupos. Encontrou-se que $75 \%$ dos grupos localiza-se em quatro dos 32 Estados do país e que $90 \%$ pertence a instituições de educação superior. Há diversidade enquanto aos interesses de pesquisa e destacam-se quatro linhas principais: energia e potência, gestão energética, hidrocarburos e energias renováveis. A produção científica está centrada basicamente em artigos científicos, livros e desenvolvimento de projetos; enquanto a patentes, os resultados são escassos. Em relação com energias renováveis, a produção científica começou a surgir a partir de 1999 e há experimentado um crescimento com tendência lineal. Logrou-se identificar que embora exista uma pesquisa estabelecida em temas de energia desde as instituições de educação superior e algumas empresas do sector energético, os avanços relacionados com energias renováveis são ainda incipientes; há um caminho por percorrer, o qual tem sido abonado com as novas regulamentações que se tem dado no país.

Palavras chave: energia; energias renováveis; grupos de pesquisa; produção científica.

\section{Para citar este artículo:}

F. N. Jiménez-García, A. M. Restrepo-Franco, and L. F. Mulcue-Nieto, "Estado de la investigación en energía en Colombia: una mirada desde los grupos de investigación," Revista Facultad de Ingeniería, vol. 28 (52), pp. 9-26, Jul. 2019 https://doi.org/10.19053/01211129.v28.n52.2019.9651.

Esta obra está bajo licencia internacional Creative Commons Reconocimiento 4.0

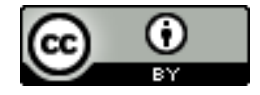




\section{INTRODUCCIÓN}

Aunque el recurso energético es un elemento vital para el desarrollo humano y la sostenibilidad de los países, su búsqueda y utilización han traído consecuencias para la sostenibilidad ambiental de nuestro planeta. La prestación del servicio energético ha tenido un fuerte impacto ambiental; en la última década, las emisiones de gases de efecto invernadero (GEI) han sido las más altas de la historia [1], a lo cual contribuye el alto uso de fuentes fósiles. En el 2014, por ejemplo, el $67 \%$ del suministro de energía eléctrica provenía de fuentes fósiles, y el $33 \%$ de fuentes no fósiles [2]. Por otra parte, pese a la importancia de este recurso, según reporta el Banco Mundial, en el 2017, 1.060 millones de personas vivían sin electricidad [3]. En el marco del acuerdo sobre cambio climático 2015, los países se comprometieron a reducir los GEI a través de la descarbonización global [4], por lo cual se han dado ciertas políticas e incentivos para acelerar los avances en la incorporación de energías renovables (ER). En los países de América Latina y el Caribe (ALC), la implementación de ER depende de la distinción entre fuentes convencionales, como la hidroeléctrica, y no convencionales, como solar, eólica, entre otras [5]. En estos países, la energía hidroeléctrica es la principal fuente de producción de electricidad (47.1\%), seguida de combustibles fósiles (42.9\%); mientras que las fuentes renovables no convencionales solo representan el $5.3 \%$ [3].

Aunque es un requisito para los países de ALC integrar una gama más amplia de tecnologías energéticas con miras a mejorar la seguridad y equidad energética y reducir la huella de carbono [2], y a pesar de las condiciones geográficas y económicas favorables, el despliegue de ER es aún lento [5]. Por tanto, la región debe diversificar la matriz energética utilizando recursos renovables como la biomasa, el viento y el sol, que complementan sus ciclos hidrológicos [6].

A fin de superar los anteriores problemas, los países se califican con el índice del trilema energético: sostenibilidad ambiental, seguridad y equidad energética [2]. Colombia ocupa la posición 46 en el mundo en el índice, y enfrenta como retos la ampliación de la cobertura, la mejora de la calidad y fiabilidad de los servicios, la 
diversificación de la matriz de energía y el sostenimiento del desarrollo económico sin aumentar las emisiones de $\mathrm{CO}_{2}[2]$.

En Colombia, la dependencia de las grandes centrales hidroeléctricas es aún mayor que en otros países, el 68.5\% de la energía total del país [3] es generada de fuentes hídricas, lo cual hace que su sistema de suministro de electricidad sea vulnerable al cambio climático y se vea afectado por fenómenos como El Niño y La Niña [7]. En cuanto a ER, en el 2014 en Colombia se promulgó la Ley 1715, que incentiva su uso [8], pero apenas en el 2018 se reglamentó dicha ley [9]; lo cual no significa que no se vinieran realizando trabajos en la incorporación y en investigaciones en torno a las ER. Es por tanto importante conocer el estado actual de la investigación en Colombia en cuanto a energía se refiere, para tener un panorama reciente y poder determinar en el futuro el impacto de las nuevas leyes en el alcance de las metas propuestas por el Protocolo de Kioto. Vale mencionar que este tipo de trabajos se realizan en otros países permanentemente [10-12].

En este trabajo se presenta el estado actual de la investigación en Colombia en temas de energía, desde la sistematización y el análisis de los avances efectuados en el país en estos temas y particularmente en ER.

\section{MATERIALES Y MÉTODOS}

En esta investigación, la cual tiene un enfoque cuantitativo y un alcance descriptivo, se hizo una recopilación y el análisis del estado actual de la investigación en Colombia en temas de energía y particularmente en ER. Para ello se llevó a cabo una búsqueda sistemática de información desde la plataforma Scienti de Colciencias, en la cual se encuentra recopilada la información de los grupos de investigación en Colombia. Se tomó una muestra de grupos de investigación inscritos en la plataforma que han declarado que realizan investigación en temas de energía.

Para la búsqueda se tuvieron en cuenta los siguientes criterios: en la plataforma Scienti, desde el ítem Servicios de Consulta, se seleccionó la opción Grupos; a continuación, Grupos por Programa Nacional de Ciencia y Tecnología; luego se escogió la opción 13, Investigaciones en Ingeniería y Minería; finalmente se 
seleccionaron los cien grupos que aparecen como fuentes primarias. Se descargó en PDF la información de estos grupos con fecha de 15 de enero de 2018 y se procedió a la recopilación y sistematización de la información de cada grupo empleando el software Excel. Posteriormente se analizó la información en forma cuantitativa, considerando tres categorías: clasificación, intereses investigativos y producción científica de los grupos.

\section{Resultados}

Los resultados se presentan en tres secciones: primera, aspectos relacionados con la conformación, clasificación y ubicación geográfica de los grupos de investigación; segunda, los intereses investigativos declarados por los grupos; tercera, la producción científica de los grupos y, particularmente, lo relacionado con ER.

\section{A. Los grupos de investigación, su ubicación, conformación y clasificación}

En primer lugar, se analizó la conformación de los grupos de investigación en el país, de acuerdo con su ubicación geográfica, el tipo de institución que los avala, el año de formación y su clasificación en Colciencias.

1) Ubicación geográfica. En cuanto a la ubicación geográfica de los grupos de investigación en energía analizados, se halló que el $75 \%$ está concentrado en cuatro departamentos del país: Antioquía, Cundinamarca, Santander y Valle del Cauca. Lo anterior puede deberse a la privilegiada ubicación geográfica de tales departamentos, lo cual los hace beneficiaros de fuentes hidrográficas importantes; y a que en estos departamentos se encuentran importantes universidades, a las que pertenecen tales grupos: Universidad de Antioquia, Universidad Nacional de Colombia, Universidad Industrial de Santander y Universidad del Valle, pioneras en la investigación en Colombia.

En el departamento de Antioquia hay alrededor de 20 empresas que se dedican a la generación, transmisión, distribución o comercialización de energía [13]; por ejemplo: ISAGEN S.A., que genera el $17 \%$ de energía eléctrica del país y comercializa el $7 \%$, y Empresas Públicas de Medellín S.A. (EPM), que genera el 22 $\%$ y comercializa el $23 \%$. Este departamento también cuenta con hidroeléctricas y 
represas de gran envergadura, como la Central Hidroeléctrica San Carlos, que es la de mayor capacidad instalada en Colombia (6020 MW) y de propiedad de ISAGEN S.A. E.S.P. Vale la pena señalar que el proyecto hidroeléctrico más grande de Colombia, que aún se encuentra en construcción, es Hidroituango, localizado sobre el río Cauca, en el noroccidente del departamento de Antioquia y ocupa predios de los municipios de Ituango y Briceño.

Por otra parte, el departamento de Cundinamarca, que se ubica en la región Centro, tiene un alto grado de industrialización y una significativa capacidad para la generación de energía eléctrica, allí se encuentra la capital del país. Existe un grupo de alrededor de 40 empresas en este departamento que se dedican a la generación, transmisión, distribución o comercialización del suministro eléctrico [13]; entre ellas está Codensa S.A. E.S.P., líder en el mercado nacional con el $24 \%$ de la participación total, que tiene presencia en Bogotá, en 97 municipios de Cundinamarca, ocho municipios de Boyacá y uno de Tolima. En Cundinamarca se encuentra una de las hidroeléctricas y represas más grandes del país, la central hidroeléctrica del Guavio, en la cuenca media-baja del río Guavio, con afluentes de los ríos Guavio, Batatas y Chivor.

En la región Oriente —donde se ubican los departamentos de Santander y Boyacá- están importantes centrales hidroeléctricas pertenecientes al Sistema Interconectado Nacional (SIN): Sogamoso y Chivor. Es importante señalar que el sector secundario en Santander es la refinación de petróleo y la industria petroquímica, lo cual lo hace un departamento fuerte en temas energéticos. En Santander se encuentran empresas como Electrificadora de Santander S.A. (de capital mixto, filial del Grupo Empresarial EPM), que se dedica a la prestación de los servicios públicos de generación, distribución, transmisión, comercialización de energía y actividades conexas en 87 municipios de Santander; además, dos empresas de Bolívar, cuatro del sur del Cesar y una de Norte de Santander. En Boyacá hay más de nueve empresas que se encargan de generar, transmitir, distribuir y comercializar la energía de la región, entre ellas la Empresa de Energía de Boyacá, que distribuye y comercializa electricidad a los 122 municipios de su región y dos municipios de Santander. 
En el Valle del Cauca existen alrededor de 26 empresas que se dedican a la generación, transmisión, distribución y comercialización de la energía en toda la región. Una de las más importantes es la Empresa de Energía del Pacífico S.A., la cual cuenta con 84 subestaciones de distribución y 16 de transmisión, que permiten atender a más de 600.000 personas en 39 municipios del Valle y uno en Chocó [13]. Además, este departamento tiene represas generadoras de energía como Anchicayá y Calima.

2) Ubicación por tipo de institución. El $90 \%$ de los grupos de investigación analizados pertenece a entidades de educación superior, mientras que solo un 10 \% está vinculado a corporaciones o institutos empresariales. Lo anterior coincide con el sistema de investigación en Colombia que, en general, aún sigue muy unido a la academia. Hay que resaltar que la Universidad Nacional, con sedes en las principales ciudades del país, es pionera en investigación, con un total de 28 grupos de los 90 mencionados, seguida de la Universidad Industrial de Santander con ocho y la Universidad de Antioquia con seis grupos. También se encuentran grupos de investigación compuestos por dos o más universidades. Hay diez grupos pertenecientes a instituciones o corporaciones empresariales, los cuales están dedicados a la investigación y desarrollo tecnológico del país. El Instituto Colombiano del Petróleo tiene cuatro grupos de investigación, y las demás corporaciones o empresas tienen uno.

3) Clasificación de acuerdo con Colciencias. El sistema de investigación colombiano divide los grupos de investigación en categorías: $A 1, A, B, C$ y $D$, donde $A 1$ es el mayor escalafón y $D$ el menor. Los grupos $A 1, A, B, C$ deben tener al menos un investigador sénior o asociado y estudiantes de doctorado como integrantes, productos de actividades relacionadas con la formación del recurso humano, de apropiación social del conocimiento y productos tipo TOP. Los A1 y A deben tener al menos cinco años de existencia, los $\mathrm{B}$ al menos tres años y los $\mathrm{C}$ como mínimo dos años; los grupos $D$ solo deben tener un año de existencia y un índice de producción de nuevo conocimiento distinto de cero [15]. De acuerdo con este escalafón, los grupos en energía se clasifican como se muestra en la Figura 1. Allí 
se observa que un $40 \%$ está ubicado en los escalafones superiores, lo cual indica que tienen una trayectoria investigativa tanto en tiempo como en producción.

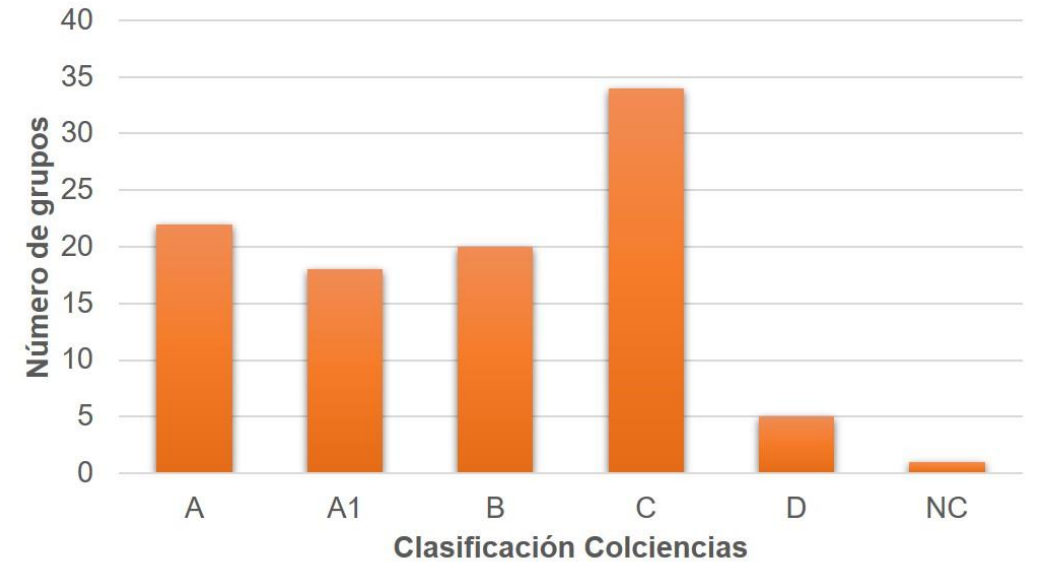

Fig. 1. Clasificación de los grupos de investigación según la categorización de Colciencias.

4) Clasificación según los años de formación. Los intervalos de tiempo de mayor aparición de grupos están entre 1995 a 2006 y 2010 a 2015, con un total de 56, tal como se muestra en la Figura 2.

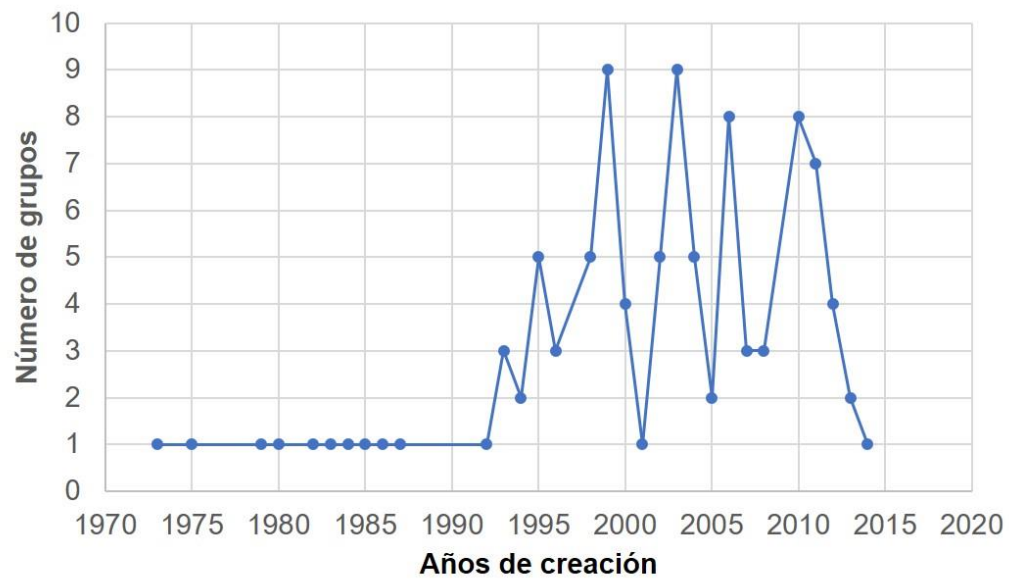

Fig. 2. Cantidad de grupos de investigación creados por año en la ventana de observación 19732017.

A comienzos de los años 90, un diagnóstico realizado a las empresas estatales de electricidad mostró resultados altamente desfavorables en términos de la eficiencia 
administrativa, operativa y financiera; además, entre 1991 y 1992 se produjo un racionamiento de energía, el más grande de la historia reciente del país. Todo esto fomentó la investigación en temas energéticos para tratar de solucionar la problemática energética nacional [16], lo cual concuerda con la concentración de creación de grupos de investigación en el primer período mencionado.

En el segundo período, del año 2010 al 2015, se crean 22 grupos de investigación, lo cual pudo deberse al crecimiento energético que se presentó en ese momento en el país. Así, por ejemplo, la generación de energía eléctrica en Colombia para el 2010 estuvo $1.6 \%$ por encima de la registrada en 2009, con una notable aparición de la energía térmica que participó en la generación del SIN hasta en un $53.3 \%$ en pleno desarrollo del fenómeno de El Niño (enero de 2010). Al comparar el año 2010 con el 2009, se observa que la generación térmica tuvo un incremento del $7.6 \%$, mientras que la generación hidráulica decreció en un $1.6 \%$ [17]. En cuanto a la demanda, en el 2011 alcanzó un crecimiento equivalente al $1.8 \%$, inferior al presentado en 2010 (2.7\%); en el 2012 el crecimiento fue del $3.8 \%$ con relación al año 2011; en el 2014 aumentó en un 4.4 \% frente al 2013, que se constituyó en el mayor incremento de demanda en los últimos diez años; y en el año 2015 creció en un $4.2 \%$ [18].

Esta evolución positiva se debió principalmente a la mejora en la demanda, debido a los altos consumos en el sector residencial, a los resultados de las altas temperaturas registradas en el país por la presencia del fenómeno de El Niño, y a la recuperación de la crisis económica [17]. Cabe resaltar que en esos años, en algunos meses se registraron bajos consumos y uno de los aspectos causantes fueron las bajas temperaturas registradas en el país por la presencia del fenómeno de La Niña, lo cual se ve reflejado en el comportamiento de la demanda del mercado regulado [18]. Las condiciones climáticas son de gran impacto en el sector energético del país, por ser protagonistas a la hora de generar y comercializar la energía. 


\section{B. Intereses investigativos de los grupos}

La segunda categoría de análisis está relacionada con los intereses investigativos de los grupos, desde las 461 líneas de investigación que declaran. En la Tabla 1 se exponen las líneas de interés y los porcentajes de ubicación de los grupos en cada una. Hay, en promedio, entre 4 y 5 líneas por grupos.

Tabla 1. Líneas de investigación más significativas, breve descripción y porcentaje de grupos que las declaran.

\begin{tabular}{|c|c|c|}
\hline Línea & Temas de la línea o sublíneas & $\begin{array}{c}\text { Grupo/ } \\
\text { Línea }\end{array}$ \\
\hline $\begin{array}{l}\text { Eficiencia } \\
\text { energética }\end{array}$ & $\begin{array}{l}\text { Eficiencia energética y diseños de procesos energéticamente } \\
\text { eficientes. }\end{array}$ & $3 \%$ \\
\hline Biocombustibles & $\begin{array}{l}\text { Biocombustibles a partir del aceite de palma, aprovechamiento } \\
\text { energético de biocombustibles, coproductos, análisis energético y } \\
\text { exergético, microalgas y producción de biogás. }\end{array}$ & $3.9 \%$ \\
\hline Minería & $\begin{array}{l}\text { Optimización minera, valoración del riesgo y regulación minera, } \\
\text { beneficio de minerales y carbones, geología, procesamiento de } \\
\text { minerales, metalurgia, desarrollo sostenible y medio ambiente y } \\
\text { minería, procesos del carbón. }\end{array}$ & $4.8 \%$ \\
\hline Materiales & $\begin{array}{l}\text { Recubrimientos, desarrollo de aplicación de materiales, } \\
\text { caracterización de materiales, biomateriales, materiales compuestos } \\
\text { y polímeros, manejo de recursos. }\end{array}$ & $8.7 \%$ \\
\hline $\begin{array}{l}\text { Energías } \\
\text { renovables }\end{array}$ & $\begin{array}{l}\text { Energía solar, energía eólica, biomasa y su transformación, } \\
\text { implementación de proyectos de ER, políticas y regulaciones y } \\
\text { enseñanza de ER en el país. }\end{array}$ & $8.9 \%$ \\
\hline Hidrocarburos & $\begin{array}{l}\text { Modelación y simulación geoestadística de yacimientos, catalizadores } \\
\text { para la conversión de hidrocarburos, reducción del impacto ambiental } \\
\text { generado por el uso combustibles fósiles, reactividad y conversión de } \\
\text { combustibles, caracterización de yacimientos, aspectos } \\
\text { geomecánicos de la ingeniería de yacimientos, regulación petrolera, } \\
\text { optimización de procesos en el sector petrolero. }\end{array}$ & $10 \%$ \\
\hline $\begin{array}{l}\text { Energía y } \\
\text { potencia }\end{array}$ & $\begin{array}{l}\text { Sistemas y calidad de potencia, conversión de energía, análisis de } \\
\text { sistemas eléctricos, monitoreo, control, optimización de sistemas de } \\
\text { potencia, generación, transmisión y distribución energética. }\end{array}$ & $13.2 \%$ \\
\hline $\begin{array}{l}\text { Gestión } \\
\text { energética }\end{array}$ & $\begin{array}{l}\text { Economía energética y regulación, mercados de energía, política } \\
\text { energética, recursos energéticos y medio ambiente, optimización y } \\
\text { uso racional de energía. }\end{array}$ & $13.4 \%$ \\
\hline Otras & $\begin{array}{l}\text { Desarrollo tecnológico, gestión de riesgos, transporte, corrosión, } \\
\text { manejo de residuos, carreteras inteligentes, robótica y automatización, } \\
\text { telecomunicaciones, mecánica de fluidos, investigación de } \\
\text { operaciones, contaminación, control ambiental, política y estrategia, } \\
\text { movilidad sostenible, calidad de aire y emisiones atmosféricas, etc. }\end{array}$ & $34.1 \%$ \\
\hline
\end{tabular}

Es importante anotar que 40 grupos reportan como línea de investigación las ER, pero hay 24 más que, aunque no declaran esta línea de investigación, sí cuentan con productos en estos temas. Esto puede deberse a que en el momento de la 
creación del grupo no tenían contemplada esta línea, pero han ido incorporándola a sus investigaciones debido a las necesidades del medio.

\section{Producción científica de los grupos}

La tercera categoría tiene que ver con la producción científica de los grupos, la cual se analiza desde tres aspectos: producción científica en general, publicación de artículos científicos y trabajos de grado dirigidos.

1) Producción científica en general. La plataforma Scienti de Colciencias divide la producción de los grupos de investigación en artículos científicos, patentes, libros, capítulos de libros, proyectos, software, prototipos, eventos científicos y trabajos dirigidos divididos en tutorías, monografías, trabajos de grado de pregrado, trabajos de grado de maestría o especialización médica, y tesis de doctorados. En la Tabla 2 se describe el total de producción científica de los grupos de investigación en energía. Es notable su producción científica en temas en general, sin embargo, en ER es baja en comparación con el total. Es de anotar que solo dos grupos de investigación han patentado equipos afines con las energías renovables, los cuales son: secador híbrido solar-biomasa para plantas aromáticas, secador solar indirecto de tiro forzado y destilador solar de dos vertientes.

Tabla 2. Producción científica de los grupos de investigación en la ventana 1973-2017.

\begin{tabular}{|l|c|c|c|c|}
\hline \multicolumn{1}{|c|}{ Tipo de producción } & $\begin{array}{c}\text { N.o de } \\
\text { grupos }\end{array}$ & $\begin{array}{c}\text { Producción } \\
\text { total }\end{array}$ & $\begin{array}{c}\text { N.o grupos en } \\
\text { ER }\end{array}$ & $\begin{array}{c}\text { Producción } \\
\text { en ER }\end{array}$ \\
\hline Artículos científicos & 100 & 7874 & 58 & 348 \\
\hline Patentes & 14 & 14 & 2 & 3 \\
\hline Libros & 73 & 520 & 26 & 69 \\
\hline Capítulos de libros & 66 & 565 & 24 & 69 \\
\hline Proyectos & 93 & 3761 & 55 & 270 \\
\hline Software & 56 & 500 & --- & --- \\
\hline Prototipos & 49 & 352 & --- & --- \\
\hline Eventos científicos & 99 & 3803 & --- & --- \\
\hline
\end{tabular}

2) Publicación de artículos científicos. En promedio, hay 78 artículos por grupo y una producción de aproximadamente 192 artículos por cada año; la publicación en ER es de $4.43 \%$ de la producción total y en ella han participado 58 grupos. La 
tendencia de publicación de los grupos en temas relacionados con ER inició en 1999 con la publicación de un artículo; esta tendencia es creciente, llegando a un máximo de 39 artículos publicados en el año 2015 (Figura 3).

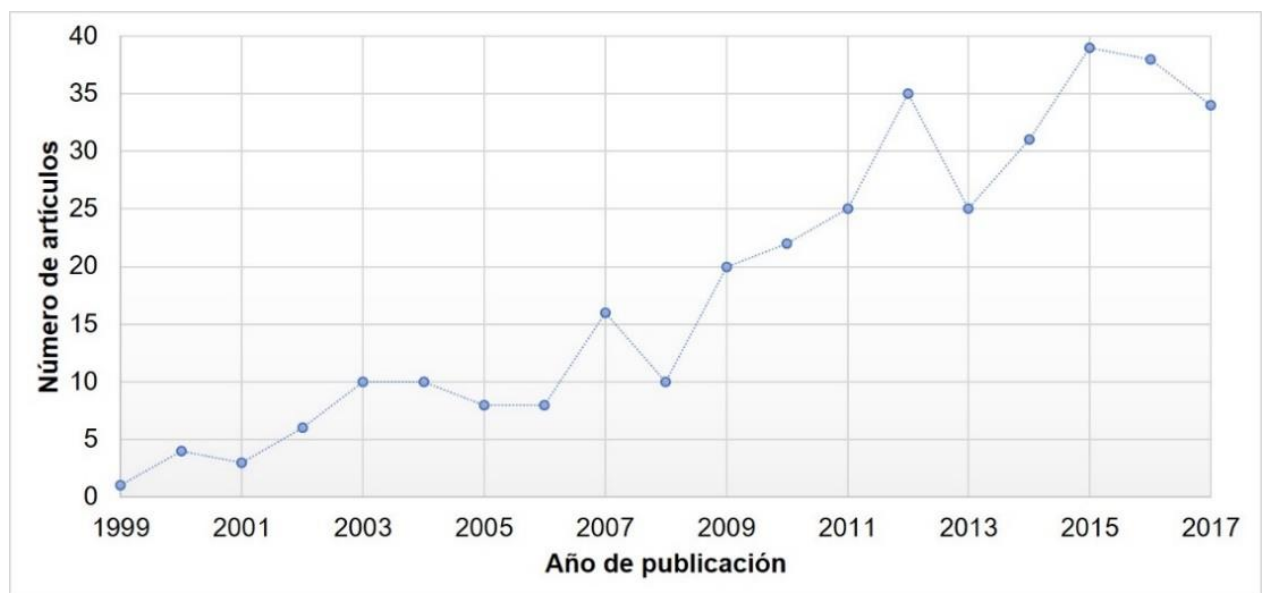

Fig. 3. Número de artículos publicados por los grupos, relacionados con ER, en la ventana 1999 2017.

Los 348 artículos publicados en ER se dividen en las siguientes sublíneas: biomasa $42.8 \%$, energía solar $37.9 \%$, políticas y gestión energética $14.1 \%$, recurso energético renovable $13.8 \%$, energía eólica $10.1 \%$, implementación de energías renovables y enseñanza de las energías renovables $2.3 \%$. En la Figura 4 se muestra el número total de artículos publicados en temas de cada sublínea, donde es notable una mayor producción en biomasa y energía solar. En cuanto a la indexación de las revistas en las cuales se han publicado los artículos en ER, la mayor participación está en revistas de carácter nacional, pero también hay presencia de publicaciones en el ámbito internacional.

Como puede observarse en el esquema presentado en la Figura 4, de los 348 artículos publicados en ER, el 46.3 \% (161) se encuentra en la base de indexación de Scopus, la cual es medida con el indicador SJR (Scimago Journal Rank) que permite ordenar las revistas de un campo disciplinar específico en orden descendente a manera de ranking [19]. Este ranking se basa en cuartiles que expresan la distribución según los valores del indicador en cuatro partes iguales, 
puede decirse que un porcentaje considerable de estos artículos está en el cuartil más alto (Q1) al momento de su publicación.

3) Trabajos de grado dirigidos. En cuanto a trabajos de grado dirigidos por estos grupos, y particularmente en temas relacionados con ER, hay 552 trabajos de pregrado, dos en especializaciones, 161 trabajos de maestría, 21 tesis doctorado y 55 en la categoría otros, la cual hace referencia a monografías, iniciación científica o semilleros. Es decir, hay un $69.4 \%$ de trabajos en estudios de pregrado frente a un $20.3 \%$ de maestría y solo un $3 \%$ en doctorado.

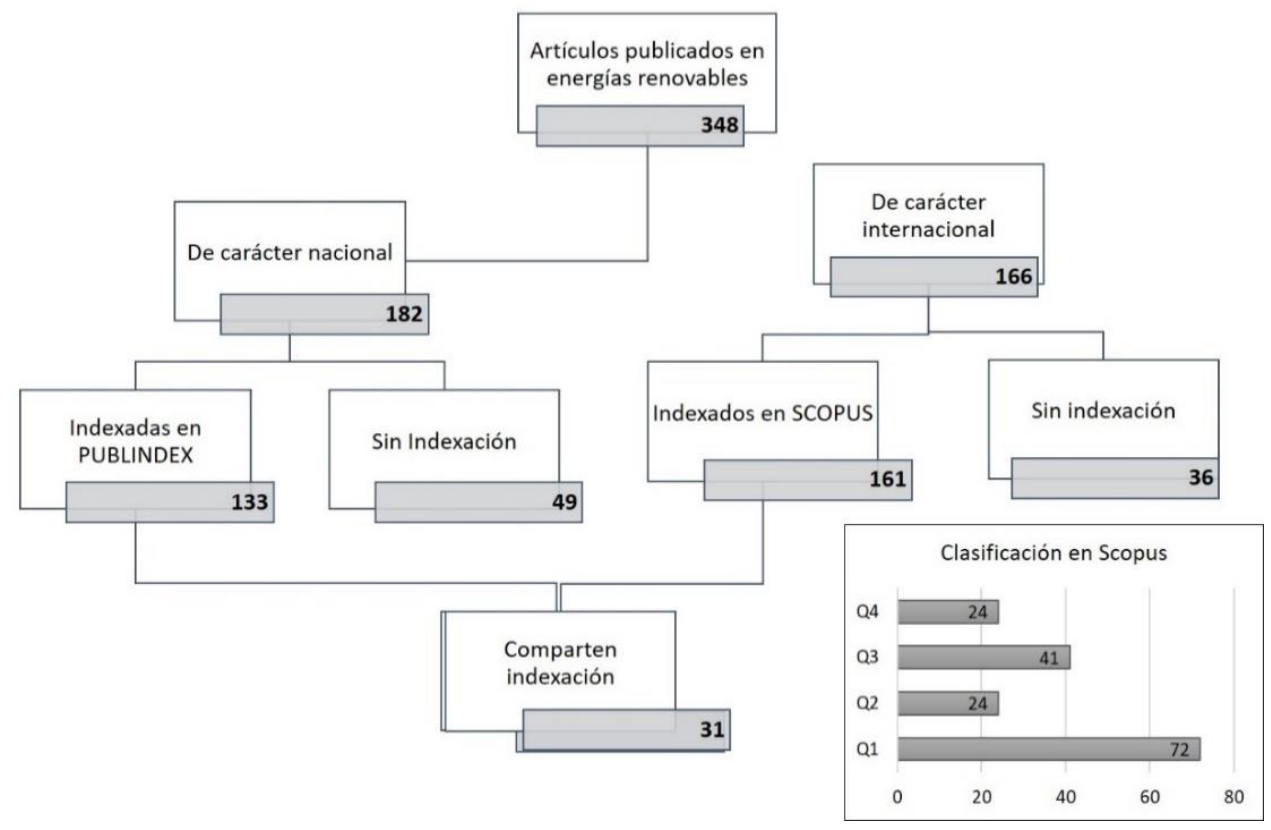

Fig. 4. Esquema con la distribución de artículos publicados en ER de acuerdo con su indexación nacional e internacional.

Hay 21 tesis de doctorado reportadas relacionadas con ER, cerca del $48 \%$ de estas tesis se refiere a energía solar (y de estas, el $70 \%$ trata sobre materiales para el desarrollo de celdas solares), un $29 \%$, con biomasa, y un $19 \%$, con energía eólica. Una de las tesis (4\%) versa sobre las ER en general. De estos trabajos, nueve han sido realizados en la Universidad de Antioquia, tres en la Universidad Nacional Sede Bogotá, cinco en la Universidad Nacional Sede Medellín, dos en la Universidad del Valle, y dos en la Universidad Pontificia Bolivariana. El período de terminación de estas tesis está comprendido entre 2006 y 2017 con los siguientes resultados: en el 
2012 había cinco trabajos terminados, en 2011 y 2014, tres, en 2009, 2010 y 2015, dos, y en 2006, 2013, 2016 y 2017 , uno.

\section{DISCUSIÓN Y CONCLUSIONES}

Se evidencian dos momentos de evolución de la investigación en energía en Colombia; uno de ellos se dio en 1992, posterior al racionamiento energético que sufrió el país: y el otro, en 2010, después de un fuerte período de fenómeno de El Niño. Es claro que las necesidades energéticas del país han llevado a impulsar el trabajo de los grupos de investigación.

Todos los resultados presentados en este trabajo, en los que pueden verse los intereses investigativos y la producción académica de los grupos de investigación en Colombia en temas energéticos en los últimos 20 años, unidos a las políticas nacionales que incentiva la incorporación de ER al sistema energético nacional, permiten concluir que el panorama investigativo en Colombia, en relación con ER, es prometedor para un futuro cercano. Si bien los grupos de investigación han venido aumentando su producción de artículos científicos a lo largo de los últimos 20 años en temas relacionados con ER, otros aspectos referentes a producción científica, como la generación de patentes, son aún incipientes.

La plataforma Scienti es una herramienta adecuada y fiable para extraer la información, ya que cada convocatoria de Colciencias obliga a los grupos e investigadores del país a realizar las actualizaciones en forma permanente; además, las últimas convocatorias exigen una supervisión de la información allí almacenada por parte de las instituciones, la cual puede ser verificada en cualquier momento por Colciencias. Se debe mencionar que algunos grupos que no se encuentran registrados en la categoría de energía también llevan a cabo trabajos en el área, aun perteneciendo a otro tema, pero estos no han sido tenidos en cuenta en este análisis debido a la dificultad de encontrarlos de forma sistemática en la plataforma.

El presente trabajo se convierte en una línea base para poder determinar en el futuro el impacto de las políticas y reglamentaciones que se vienen dando en el país en torno a ER, pues se espera que estas políticas potencien tanto la implementación 
de nuevas fuentes de energía, así como su investigación. Para trabajos futuros se podrían complementar los resultados con los que se pueden obtener de otras plataformas como los repositorios institucionales, aunque este trabajo sería evidentemente más dispendioso.

\section{CONTRIBUCIÓN DE LOS AUTORES}

La coordinación del trabajo y escritura del documento lo realizó Francy Nelly Jiménez, la recopilación y organización de la información fue llevada a cabo por Alejandra María Restrepo y Luis Fernando Mulcue apoyo en el proceso de análisis de la información.

\section{AgRADECIMIENTOS}

Los autores agraden a la Universidad Autónoma de Manizales, por su apoyo al proyecto de investigación inscrito en la Unidad de Investigación de la institución, identificado con código 565-082, y a Colciencias, por su apoyo a través de la Convocatoria 775 de 2017 para jóvenes investigadores e innovadores por la paz.

\section{REFERENCIAS}

[1] The Intergovernmental Panel on Climate Change -IPCC-, Renewable Energy Sources and Climate Change Mitigation. Special Report of the Intergovernmental Panel on Climate Change, 2012. Available at: https://www.ipcc.ch/site/assets/uploads/2018/03/Title-1.pdf.

[2] Consejo Mundial de la Energía, Consejo Mundial de la Energía, 2017. Available at: https://www.worldenergy.org/wp-

content/uploads/2014/04/WEC 16 page document 21.3.14 ES FINAL.pdf.

[3] Unidad de Relaciones Externas e Institucionales del Grupo Banco Mundial, Informe anual: Poner fin a la pobreza. Invertir para generar oportunidades, 2013. Available at: http://www.bancomundial.org/es/results/2013/04/13/climate-change-results-profile.

[4] C. García Arbeláez, X. Barrera, R. Gómez, and R. Suárez Castaño, El ABC de los compromisos de Colombia para la COP21. Colombia: WWF-Colombia, 2015.

[5] D. Jacobs, N. Marzolf, J. Paredes, W. Rickerson, H. Flynn, B. Becker, and P. Solano, "Analysis of Renewable Energy Incentives in the Latin America and Caribbean Region: The Feed-in Tariff Case," Energy Policy, vol. 60, pp.601-610, Sep. 2013. https://doi.org/10.1016/j.enpol.2012.09.024.

[6] N. Nuttall, Informe anual. División de Comunicaciones e Información Pública del Programa de las Naciones Unidas para el Desarrollo Medioambiental, 2011.

[7] W. Cai, G. Santoso, S. Yeh, S. An, K. Cobb, M. Collins, F. Jin, J. Kug, M. Lengaigne, M. Mcphaden, K. Takahashl, A. Timmermann, G. Vecchl, M. Watanabe, and L. Wu, "ENSO and Greenhouse Warming," 
Francy Nelly Jiménez-García; Alejandra María Restrepo-Franco; Luis Fernando Mulcue-Nieto

Nature Publishing Group, vol. 5 (9), pp. 849-859, 2015. https://doi.org/10.1038/nclimate2743.

[8] Congreso de la República de Colombia, Ley 1715 de 2014, 2014 . Available at: http://www.secretariasenado.gov.co/senado/basedoc/ley 1715 2014.html.

[9] Ministerio de Minas y Energía, Resolución 030 de 2018, 2018. Available at: http://apolo.creg.gov.co/Publicac.nsf/1c09d18d2d5ffb5b05256eee00709c02/83b41035c2c4474f05258 243005a1191? OpenDocument.

[10] F. Romo, B. Guerrero, and A. Moya, "Análisis de la producción científica española en energías renovables, sostenibilidad y medio ambiente (Scopus, 2003-2009) en el contexto mundial," Investigación Bibliotecológica, vol. 27 (60), pp 125-151, 2013. https://doi.org/10.1016/S0187358X(13)72546-2.

[11] A. Endo, I. Tsurita, K. Burnett, and P. M. Orencio, "A Review of the Current State of Research on the Water, Energy, and Food Nexus," Journal of Hydrology Regional Studies, vol. 11, pp. 20-30, Jun. 2017. https://doi.org/10.1016/j.ejrh.2015.11.010.

[12] A. Celaya Lozano, D. Luque Agraz, J. García Hernández, J. A. Amozurrutia de María y Campos, J. M. Preciado Rodríguez, J. Laborín Álvarez, and R. E. Cabanillas López, "Evaluación de la producción científica de sustentabilidad ambiental en un centro público de investigación (cpi) del Conacyt (19822012)", Rev. la Educ. Super., vol. $46 \quad$ (182), pp. 89-112, 2017. https://doi.org/10.1016/j.resu.2017.04.002.

[13] R. A. Palacios, Inventario documentado de represas en Colombia, Thesis, Universidad Militar, Bogotá D. $\quad$ C., $2013 . \quad$ Available at: https://repository.unimilitar.edu.co/bitstream/10654/11360/1/PalaciosSierraRicardoAndres2013.pdf.

[14] Departamento Administrativo de Ciencia Tecnología e innovación COLCIENCIAS, Modelo de medición grupos de investigación, desarrollo tecnológico o de innovación y de reconocimiento de investigadores del sistema nacional de ciencia, tecnología e innovación, 2015. Available at: http://www.colciencias.gov.co/sites/default/files/upload/noticias/mediciondegrupos-actene2015.pdf.

[15] Comisión de Regulación de Energía y Gas -CRE-, Quienes somos, 2018. Available at: http://www.creg.gov.co/index.php/creg/quienes-somos/historia.

[16] Ministerio de Minas y Energía, El futuro es de todos, 2011 . Available at: https://www.minminas.gov.co/documents/10180/23400/05-ENERGIA2010-2011.pdf/770a198d-d4ee4687-b74c-74592b403ae6.

[17] XM. Filial de ISA, Informe de operación del SIN y administración del mercado, 2014. Available at: http://informesanuales.xm.com.co/2014/SitePages/operacion/3-1-Demanda-de-energianacional.aspx.

[18] B. González-Pereira, V. P. Guerrero-Bote, and F. Moya-Anegón, "A New Approach to the Metric of Journals? Scientific Prestige: The SJR Indicator," Journal of Informetrics, vol. 3, pp. 379-391, Jul. 2010. https://doi.org/10.1016/j.joi.2010.03.002.

Revista Facultad de Ingeniería (Rev. Fac. Ing.) Vol. 28 (52), pp. 9-26. Julio-Septiembre 2019. Tunja-Boyacá, Colombia. L-ISSN: 0121-1129, e-ISSN: 2357-5328, DOI: https://doi.org/10.19053/01211129.v28.n52.2019.9651 